\title{
Effect of Two Anesthetic Regimes with Dexmedetomidine as Adjuvant on Transcranial Electrical Motor Evoked Potentials during Spine Surgery
}

\author{
Rajeeb K. Mishra ${ }^{1}$ Hemanshu Prabhakar ${ }^{2}$ Indu Kapoor ${ }^{2}$ Dinu S. Chandran ${ }^{3} \quad$ Arvind Chaturvedi $^{2}$
}

1Department of Neuroanaesthesiology and Neurocritical Care, National Institute of Mental Health and Neurosciences, Bengaluru, Karnataka, India

${ }^{2}$ Department of Neuroanaesthesiology and Critical Care, All India Institute of Medical Sciences, New Delhi, India

${ }^{3}$ Department of Physiology, All India Institute of Medical Sciences, New Delhi, India

J Neuroanaesthesiol Crit Care 2020;7:84-90

\author{
Address for correspondence Indu Kapoor, MD, Department of \\ Neuroanaesthesiology and Critical Care, Neurosciences Centre, \\ All India Institute of Medical Sciences, New Delhi 110029, India \\ (e-mail: dr.indu.me@gmail.com).
}

\begin{abstract}
Keywords

- dexmedetomidine

- propofol

- desflurane

- transcranial electrical motor evoked potential

- amplitude

- spine surgery

Background Transcranial motor evoked potential (TCMEP) recording during spinal cord/spinal column surgery is a reliable and valid diagnostic adjunct to assess spinal cord integrity and is recommended if utilized for this purpose. Electrophysiologic monitoring in terms of TcMEP has been proven to be a useful tool in detecting spinal cord dysfunction at the earliest and allows corrective action to be taken before permanent neuronal dysfunction sets in. The quality of intraoperative neuromonitoring is influenced by various factors. Most anesthetics used in clinical practice suppress the evoked potentials. Thus, selecting an appropriate technique is always a challenging task.

Materials and Methods Thirty ASA I and II patients scheduled for elective dorsolumbar spine surgery with TCMEP monitoring were recruited in the study. Patients were randomized into three groups: (1) Propofol (group P) 100 to $150 \mu \mathrm{g} / \mathrm{kg} / \mathrm{min}$ with dexmedetomidine $0.6 \mu \mathrm{g} / \mathrm{kg} / \mathrm{hr}$ and fentanyl $1 \mu \mathrm{g} / \mathrm{kg} / \mathrm{hr}$, (2) desflurane (group D) ( $<0.5 \mathrm{MAC}$ ) with dexmedetomidine $0.6 \mu \mathrm{g} / \mathrm{kg} / \mathrm{hr}$ and fentanyl $1 \mu \mathrm{g} / \mathrm{kg} / \mathrm{hr}$, and (3) standard group (group S) patients received propofol 100 to $150 \mu \mathrm{g} / \mathrm{kg} / \mathrm{min}$, fentanyl $1 \mu \mathrm{g} / \mathrm{kg} / \mathrm{hr}$ along with equal volume of saline (placebo). TcMEP amplitudes were recorded bilaterally from electrodes placed at least in one set of muscles with motor origin rostral and one set of muscle caudal to the spinal level of lesion at different time points.

Results Three patients were excluded after allocation; 27 out of 30 patients were analyzed. The demographic and surgical characteristics of patients were comparable. The stimulation voltage needed to elicit the responses in all the three groups was comparable. No difference was observed in brachioradialis muscle amplitudes between the groups at different time points. However, in the right brachioradialis muscle, we found reduced amplitudes at baseline in group D and at 120 minutes in
\end{abstract}

DOI https://doi.org/ $10.1055 / \mathrm{s}-0039-3400549$ ISSN 2348-0548.
(C)2020 Indian Society of Neuroanaesthesiology and Critical Care
License terms

(ㅇ) (1) $\ominus \circledast$ 
group P. We noticed reduced amplitudes of bilateral brachioradialis muscle in group P at 60 minutes and 90 minutes with respect to the baseline. For lower extremity, we measured amplitudes of TcMEP in tibialis anterior (TA) and did not find any difference in amplitudes between the groups at different time points.

Conclusion We observed that the desflurane-dexmedetomidine combination did not hinder TCMEP as compared with both standard and propofol-dexmedetomidine groups. Thus, this combined regime could be used in surgeries requiring motor evoked potential monitoring.

\section{Introduction}

Transcranial motor evoked potential (TcMEP) recording during spinal cord/spinal column surgery is a reliable and valid diagnostic adjunct to assess spinal cord integrity and is recommended if utilized for this purpose. ${ }^{1}$ Electrophysiologic monitoring in terms of TcMEP has been proven to be a useful tool in detecting spinal cord dysfunction at the earliest and allows corrective action to be taken before permanent neuronal dysfunction sets in. ${ }^{2}$ The quality of intraoperative neuromonitoring is influenced by various factors., ${ }^{3,4}$ Most anesthetics used in clinical practice suppress the evoked potentials. Thus, selecting an appropriate technique is always a challenging task. Literature suggests that the total intravenous anesthetics (TIVA) technique is better than inhalational agents. ${ }^{5-8}$ The effect of older inhalational agents is suppressive on evoked potentials. However, new inhalational agents such as sevoflurane and desflurane showed encouraging effects on evoked potentials with a lower minimum alveolar concentrations (MAC). ${ }^{9,10}$ A 0.3 MAC of desflurane provided acceptable evoked potential recording and was superior to sevoflurane in this regard. ${ }^{11}$ In contrast, another study stated that desfluarne (MAC of 0.5-0.6) could significantly decrease the motor evoked potential amplitude when compared with propofol. ${ }^{12}$ In recent years, a lot of adjuvants along with TIVA have been tried. ${ }^{13-15}$ Dexmedetomidine, an $\alpha_{2}$ agonist, has been tried because of potentiation of the hypnotic effects of propofol while reducing the dose required. Most authors suggest that addition of dexmedetomidine has no attenuating effect on evoked potentials. ${ }^{14}$ In a recent study, authors concluded that dexmedetomidine did not alter evoked potentials and could be safely administered. ${ }^{15}$ Use of propofol-based anesthesia is popularly regarded as the standard technique while considering TcMEP; however, its potential side effects cannot be ignored. ${ }^{16}$ The side effects can possibly be exaggerated in younger children and patients with autonomic neuropathy. Hence, we planned to conduct this study to suggest an alternate desflurane-based technique with low concentration to facilitate monitoring. To date, there are no studies comparing the effects of desflurane and propofol with dexmedetomidine as an adjuvant.

We hypothesized that changes in amplitudes with desflurane are equivalent to that observed in the propofol group. The main aim of our study was to compare the effect of two anesthetic regimes with dexmedetomidine as an adjuvant on amplitudes of transcranial electrical motor evoked potentials during spine surgery.

\section{Materials and Methods}

Our manuscript adheres to the applicable CONSORT guidelines and the clinical trial was registered prior to patient enrolment [CTRI/2017/08/009191 on 01/08/2017].

After institutional ethics committee approval and written informed consent, 30 patients were enrolled into the study. Patients with American Society of Anaesthesiologists (ASA) physical status I, and II, and between 18 and 60 years of age scheduled for elective dorsolumbar spine surgery with TcMEP monitoring were included in the study. Preoperative neurologic deficit with grades $<3$, pre-existing myopathies, morbid obesity, history of coronary artery disease, chronic renal and hepatic insufficiency, diabetes mellitus, heart block, allergy to any drugs, seizures, and patients having pacemakers and automatic implantable cardioverter and defibrillator were excluded. In this study, patients were prospectively studied in a randomized, placebo-controlled double-blind fashion to evaluate the effect of two anesthetic regimes with dexmedetomidine as adjuvant on TcMEP during spine surgery. Patients were premedicated with glycopyrrolate $0.2 \mathrm{mg}$ via intramuscular route 30 minutes prior to surgery. All standard monitors such as noninvasive blood pressure, electrocardiogram, and pulse oximetry were applied. Bispectral index (BIS) sensors were attached to patient's forehead to monitor depth of anesthesia. After preoxygenation with $100 \%$ oxygen, general anesthesia was induced with fentanyl $2 \mu \mathrm{g} / \mathrm{kg}$ and propofol $2 \mathrm{mg} / \mathrm{kg}$. Rocuronium $0.6 \mathrm{mg} / \mathrm{kg}$ was used to facilitate tracheal intubation. The invasive arterial blood pressure and end-tidal carbon dioxide were recorded after induction. A fresh gas flow of $2 \mathrm{~L} / \mathrm{min}$ was set with air and oxygen to deliver $40 \%$ oxygen with a ventilator setting to maintain end-tidal carbon dioxide value between 35 and $45 \mathrm{~mm} \mathrm{Hg}$. Blood pressure was maintained within $20 \%$ of mean baseline values. If required, vasopressor in the form of phenylephrine boluses were given as and when required to maintain desired mean arterial blood pressure. The patients were randomized into three groups using a computer-generated randomization chart. Opaque sealed envelopes were used for group allocation. 
Patients received either propofol 100 to $150 \mu \mathrm{g} / \mathrm{kg} / \mathrm{min}$ (group P) or desflurane $(\leq 0.5 \mathrm{MAC}$ ) (group $\mathrm{D})$. In both the groups, infusions of dexmedetomidine $0.6 \mu \mathrm{g} / \mathrm{kg} / \mathrm{hr}$ and fentanyl $1 \mu \mathrm{g} / \mathrm{kg} / \mathrm{hr}$ was administered. In the standard group, patients received propofol 100 to $150 \mu \mathrm{g} / \mathrm{kg} / \mathrm{min}$ and fentanyl $1 \mu \mathrm{g} / \mathrm{kg} / \mathrm{hr}$, along with equal volume of saline (group S). BIS values were maintained within 30 to 60 in all the study groups. All infusions were stopped at the beginning of skin closure.

TcMEP amplitudes were recorded bilaterally from electrodes placed at least in one set of muscles with motor origin rostral and one set of muscle caudal to the spinal level of lesion at different time points using the NIM-Eclipse nerve monitoring system (Medtronic; Minneapolis, Minnesota, United States). The responses were elicited following transcranial stimulation of electrodes placed at $\mathrm{C}_{3}-\mathrm{C}_{4}$ using an interstimuli interval varying from 3 to 5 milliseconds, pulse duration of 50/75 microseconds, and stimulation voltages varying between 200 and $1,000 \mathrm{~V}$, train of 6 to 8 pulses. Baseline $\left(\mathrm{T}_{0}\right)$ TcMEP amplitudes were recorded before incision. Later TcMEP amplitudes were recorded every 30-minute interval for first 2 hours and were designated as $\mathrm{T}_{1}$ (at 30 minute), $\mathrm{T}_{2}$ (at 60 minute), $\mathrm{T} 3$ (at 90 minute), and T4 (at 120 minute). The values were recorded hourly thereafter till the end of surgery.

\section{Statistical Analysis}

All statistical analyses were performed using STATA 14.0 (Stata Corp; College Station, Texas, United States). Fisher exact test was used for categorical variables. For all parametric distribution of data, we applied one-way analysis of variance. For nonparametric data, such as age and TcMEP amplitudes, we used Kruskal-Wallis test for comparison. We compared the amplitudes in between groups and within groups as well. Apart from that we also did a comparison of the amplitudes at specified time points from the baseline amplitude in all groups. We also compared the amplitudes between right and left limbs at different time points to look into any differential effect of agents. A $p$-value of less than 0.05 was considered as statistically significant.

\section{Results}

A total of 30 patients were eligible for this study. Three patients ( 2 from group D, 1 from group $P$ ) were excluded after allocation; 27 out of 30 patients were analyzed (-Fig. 1). The reason for exclusion in group $\mathrm{P}$ after allocation was technical problem, which could not be corrected. In group D we could not obtain the baseline TcMEP of lower limb, and hence switched to group S; however, even after switching we did not acquire appreciable recordings.

The demographic and surgical characteristics of patients in the three groups were comparable (-Table 1). Patients in all the groups were hemodynamically stable and there was no difference between the groups in heart rate (HR). However, the mean arterial pressure (MAP) values were different between groups at 30 minutes and at 60 minutes. The difference was statistically significant when comparison made between group $\mathrm{D}$ and group $\mathrm{P}(0.04)$, also with group $\mathrm{D}$ and group $\mathrm{S}(p=0.02)$ (-Table 2 ). We maintained BIS value within 30 to 60 in all groups at all time points. It is noteworthy to mention that we did not observe burst suppression in any of our study population. However, BIS values were higher in the desflurane group, which was statistically

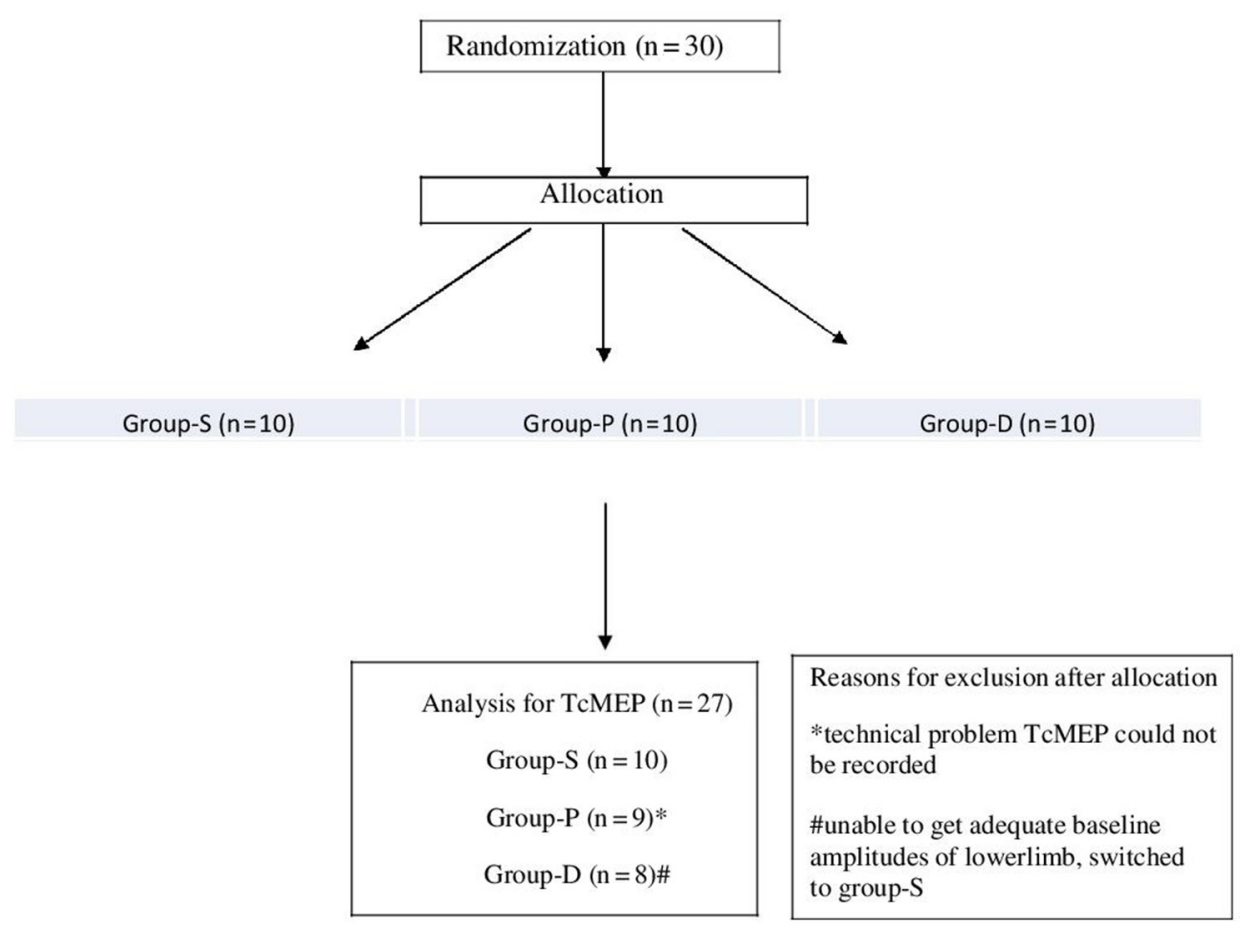

Fig. 1 Flowchart of trial and reasons for exclusion after allocation. TcMEP, transcranial motor evoked potential. 
Table 1 Comparison of patient demographics and intraoperative data (median [range], number (\%), mean + SD)

\begin{tabular}{|c|c|c|c|c|}
\hline & Group S $(n=10)$ & Group P $(n=9)$ & Group D $(n=8)$ & $p$-Value \\
\hline Age $(y)$ & $32(20-56)$ & $26(18-48)$ & $32(23-53)$ & 0.43 \\
\hline \multicolumn{5}{|l|}{ Gender } \\
\hline Male & $4(40)$ & $5(55.6)$ & $5(62.5)$ & \multirow[t]{2}{*}{0.70} \\
\hline Female & $6(60)$ & $4(44.4)$ & $3(37.5)$ & \\
\hline BMI $\left(\mathrm{kg} / \mathrm{m}^{2}\right)$ & $24.1(17.5-27.7)$ & $22.9(18.4-29)$ & $23.3(17.9-29.4)$ & 0.98 \\
\hline Duration of surgery (min) & $257.5(148-460)$ & $250(150-380)$ & $260(185-345)$ & 0.94 \\
\hline Estimated blood loss (mL) & $300(200-600)$ & $300(100-900)$ & $500(100-1200)$ & 0.85 \\
\hline Input volume (mL) & $2500(1500-5000)$ & $2500(2000-4000)$ & $3500(2000-4000)$ & 0.55 \\
\hline Urine output (mL) & $500(300-1700)$ & $750(200-1600)$ & $1000(600-1700)$ & 0.42 \\
\hline Time to extubation (min) & $15(10-23)$ & $10(5-60)$ & $10(7-20)$ & 0.44 \\
\hline
\end{tabular}

Table 2 Comparison of heart rate (HR), MAP, temperature, and bispectral index (BIS) at different time points after induction of anesthesia between groups (mean + SD, median [interquartile])

\begin{tabular}{|c|c|c|c|c|}
\hline HR & Group S $(n=10)$ & Group P $(n=9)$ & Group D $(n=8)$ & $p$-Value \\
\hline T0 & $71.7+11.21$ & $65.4+12.98$ & $67.2+10.89$ & 0.49 \\
\hline T1 & $71.6+10.30$ & $66.2+9.62$ & $65.6+10.25$ & 0.38 \\
\hline $\mathrm{T} 2$ & $75.5+10.78$ & $69.3+11.14$ & $63.3+10.30$ & 0.07 \\
\hline T3 & $77.6+10$ & $74.1+13.18$ & $74.1+15.36$ & 0.81 \\
\hline $\mathrm{T} 4$ & $82.77+11.31$ & $74.5+14.97$ & $69.7+13.53$ & 0.15 \\
\hline \multicolumn{5}{|l|}{ MAP* } \\
\hline T0 & $85.6+9.94$ & $85.5+7.97$ & $76.7+9.3$ & 0.09 \\
\hline T1 & $89.9+8.33$ & $89.11+6.95$ & $78.62+8.81$ & $0.01^{*}$ \\
\hline $\mathrm{T} 2$ & $90.5+10.4$ & $89.62+9.07$ & $78.75+7.08$ & 0.02 \\
\hline T3 & $93.4+13.4$ & $85.3+7.01$ & $79.1+14.24$ & 0.07 \\
\hline $\mathrm{T} 4$ & $88.9+10$ & $81.5+5.2$ & $81.7+8.07$ & 0.16 \\
\hline \multicolumn{5}{|l|}{ Temperature } \\
\hline T0 & $35.3+0.57$ & $35.1+0.47$ & $34.9+0.57$ & 0.34 \\
\hline $\mathrm{T} 1$ & $35.3+0.61$ & $35.1+0.44$ & $34.8+0.52$ & 0.15 \\
\hline $\mathrm{T} 2$ & $35.5+0.54$ & $35.4+0.64$ & $34.9+0.79$ & 0.19 \\
\hline T3 & $35.6+0.56$ & $35.8+0.58$ & $35.1+0.94$ & 0.17 \\
\hline T4 & $35.9+0.6$ & $35.9+0.65$ & $35.2+1$ & 0.16 \\
\hline \multicolumn{5}{|l|}{ BIS" } \\
\hline T0 & $44.2+8.24$ & $34.6+4.5$ & $50+0.89$ & $0.001^{\#}$ \\
\hline $\mathrm{T} 1$ & $41.3+3.71$ & $33.3+5.17$ & $44.3+6.18$ & 0.001 \\
\hline $\mathrm{T} 2$ & $42.7+4.92$ & $34.4+5.04$ & $46.1+5.81$ & 0.001 \\
\hline T3 & $41.9+6.45$ & $34+4.72$ & $47.2+5.3$ & 0.001 \\
\hline T4 & $42.8+7.22$ & $37.5+4.5$ & $50.1+6.22$ & 0.006 \\
\hline Stim volt (V) & $337.5(245-500)$ & $500(350-700)$ & $425(275-550)$ & 0.59 \\
\hline
\end{tabular}

Note: T0-baseline, T1-30 minutes, T2-60 minutes, T3-90 minutes, T4-120 minutes.

${ }^{*}$ MAP T1 group S vs. group $D p=0.02$, group P vs. group $D p=0.04 ;$ MAP T2 group $S$ vs. group $D p=0.03$, group P vs. group D $p=0.07$.

\#BIS T0 group S vs. group P $p=0.02$, group P vs. group $D p=0.001$; BIS T1 group S vs. group P $p=0.006$, group P vs. group D $p=0.001 ;$ BIS T2 group $S$ vs. group P $p=0.008$, group P vs. group D $p=0.001$; BIS T3 group S vs. group P $p=0.03$, group P vs. group D $p=0.001$, BIS T4 group $S$ vs. group $P p=0.02$, group $P$ vs. group $D p=0.006$.

significant between the groups at all time points and attributable to the statistical significant relationship between group $\mathrm{P}$ and group S, and group P and group D ( - Table 2). Amplitudes of TcMEP were successfully and adequately elicited in all patients in all the three groups. The stimulation voltage needed to elicit the responses in all the three groups was comparable (-Table 2 ). It is important to note that desflurane-dexmedetomidine combination resulted in considerably lower cMEP amplitudes at all time 
points in all the study muscles as compared with other groups, but it was not statistically different. For the upper extremity, we measured amplitudes of brachioradialis for TcMEP. There was no significant difference in amplitudes observed between the groups at different time points. However, at baseline in the right brachioradialis muscle we found reduced amplitudes in group $\mathrm{D}(p=0.04)$ and at 120 minutes in group $\mathrm{P}(0.03)$ (-Table 3$)$. We also compared amplitudes with respect to baseline, which showed a significant difference in group P in amplitudes on right side at 60 minutes $(p=0.01)$ and 90 minutes $(p=0.02)$. Similar results were also noted for the left side at 60 and 90 minutes (-Fig. 2). For the lower extremity, we measured amplitudes of TcMEP in the tibialis anterior (TA). No difference was observed in TA amplitudes in between groups ( - Table 3 ). There were no differences noted in TA amplitudes from baseline at different time points ( - Fig. 3 ).

\section{Discussion}

In our study, we used infusion of dexmedetomidine at a rate of $0.6 \mu \mathrm{g} / \mathrm{kg} / \mathrm{hr}$ as an adjuvant to the primary anesthetic agents. We selected a higher rate of infusion of dexmedetomidine based on two recent randomized conducted studies that suggested such doses did not hamper TcMEP recordings. Bala et al concluded that a target concentration up to $0.6 \mathrm{ng} / \mathrm{mL}$ of dexmedetomidine as an adjuvant does not alter the evoked potentials. ${ }^{14}$ Similarly, Rozet et al did not notice any changes in evoked potentials in a clinically relevant dose of dexmedetomidine. They used a loading dose of $0.6 \mu \mathrm{g} / \mathrm{kg}$ infused over 10 minutes, followed by $0.6 \mu \mathrm{g} / \mathrm{kg} / \mathrm{h}$, which did not alter the evoked potential. In another study Mahmoud et al, a suppression of TcMEP amplitudes was reported where dexmedetomidine at clinically relevant target plasma concentrations $(0.6-0.8 \mathrm{ng} / \mathrm{mL})$ was used as an adjuvant to propofol, which was performed with a one-time point analysis with loading of dexmedetomidine. Hence in our study, we did not use the loading dose of dexmedetomidine. ${ }^{17}$ Threshold criterion, amplitude criterion, and all or nothing criterion are generally used when TcMEP is recorded to quantify the significant neurologic injury. Guidelines suggest that loss of 50 to $100 \%$ amplitude of the motor evoked potential amplitude triggers the alarm..$^{18}$ Therefore, in our study we had decided to monitor the amplitude of evoked potential, and $80 \%$ amplitude loss was set as a warning sign of significant neurologic deficit.

Table 3 Comparison amplitudes of motor evoked potentials at different time points (median [interquartile range])

\begin{tabular}{|c|c|c|c|c|}
\hline Upper limb & Group S $(n=10)$ & Group P $(n=9)$ & Group D $(n=8)$ & $p$-Value \\
\hline \multicolumn{5}{|l|}{ Right } \\
\hline T0 & $1143.5(478-1983)$ & $2014(434-3030)$ & $457.5(242-602)$ & 0.04 \\
\hline T1 & $1572(1016-1786)$ & $869(460-3088)$ & $482.5(305-698)$ & 0.11 \\
\hline $\mathrm{T} 2$ & $1352(1152-2594)$ & $663.5(383-1885)$ & 587 (286.5-929) & 0.10 \\
\hline T3 & $1659(1392-2260)$ & $432(282-2298)$ & $573(345.5-883)$ & 0.21 \\
\hline T4 & $1706(564-3466)$ & 380.5 (284-469) & $610(375-1120)$ & 0.03 \\
\hline \multicolumn{5}{|l|}{ Left } \\
\hline T0 & 967 (372-1458) & 709 (220-2838) & $374(216-662)$ & 0.28 \\
\hline $\mathrm{T} 1$ & $819(518-1602)$ & $659(256-2360)$ & $359.5(262-592)$ & 0.22 \\
\hline $\mathrm{T} 2$ & $1003.5(582-2044)$ & $351.5(160.5-1340)$ & $395(321.5-1518)$ & 0.12 \\
\hline T3 & $996(534-1942)$ & $522(214-1599)$ & $392(292-1625)$ & 0.31 \\
\hline $\mathrm{T} 4$ & $936(571-2322)$ & $399.5(246-780)$ & $418(292-1386)$ & 0.35 \\
\hline \multicolumn{5}{|l|}{ Lower limb } \\
\hline \multicolumn{5}{|l|}{ Right } \\
\hline T0 & $862(404-1114)$ & $587(279-1124)$ & $361(264-764)$ & 0.51 \\
\hline $\mathrm{T} 1$ & $608(512-2234)$ & $473.5(104-1127)$ & $688(244-1096)$ & 0.77 \\
\hline $\mathrm{T} 2$ & $954(554-1620)$ & $975(82-1444)$ & $594(460-975)$ & 0.56 \\
\hline T3 & 1859 (910-2397.5) & $1080(116-1368)$ & $886(89-1456)$ & 0.48 \\
\hline T4 & 983.5 (934-2327) & 967 (60-990) & $852(93-1108)$ & 0.52 \\
\hline \multicolumn{5}{|l|}{ Left } \\
\hline T0 & $886(94-1633)$ & $608(79-1262)$ & $198.5(116-1011.5)$ & 0.77 \\
\hline $\mathrm{T} 1$ & $1014(150-2282)$ & $412(82-1450)$ & $390(90-887)$ & 0.53 \\
\hline $\mathrm{T} 2$ & $1585(121-2400)$ & $332(81.5-2168.5)$ & $284(143-968.5)$ & 0.63 \\
\hline T3 & $1780(107-2574)$ & $560(67-2958)$ & $355(177.5-682.5)$ & 0.70 \\
\hline T4 & $1692(110-2650)$ & $1280(64-2122)$ & $412(274-1308)$ & 0.75 \\
\hline
\end{tabular}

Note: T0-baseline, T1-30 minutes, T2-60 minutes, T3-90 minutes, T4-120 minutes. 

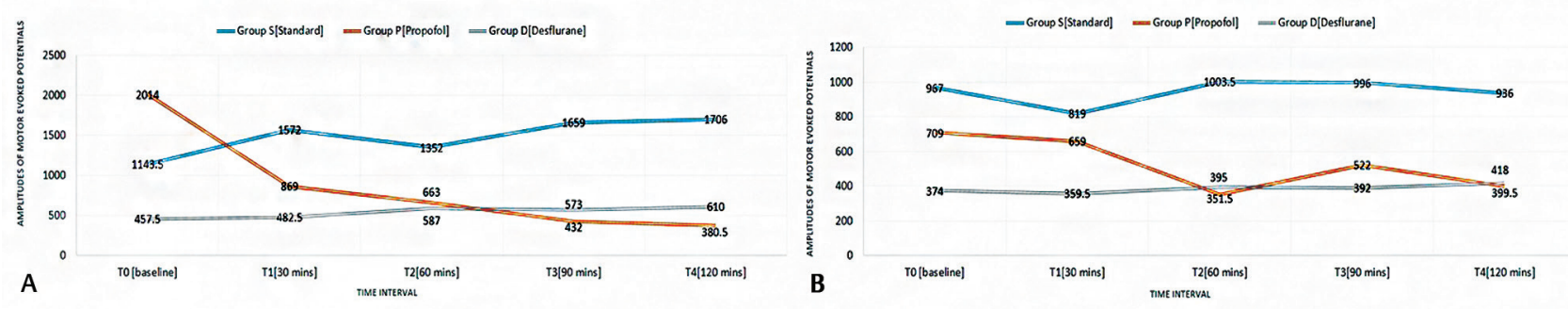

Fig. 2 Comparison of amplitudes of motor evoked potentials to baseline at different time points in the (A) right and (B) left upper limbs (median [interquartile range]).
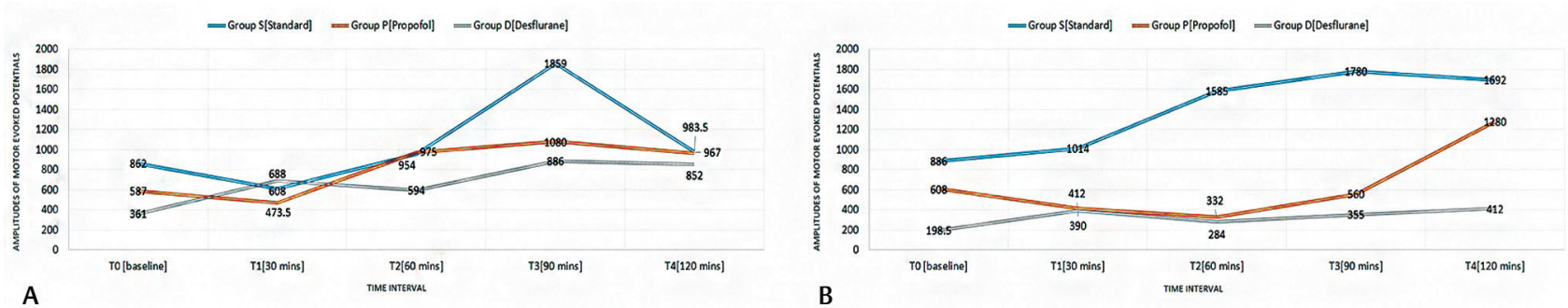

Fig. 3 Comparison of amplitudes of motor evoked potentials to baseline at different time points in the (A) right and (B) left lower limbs (median [interquartile range]).

The major difference between our study and previously published studies are that we formulated two different regimens of desflurane and propofol combined with dexmedotomidine and compared them to the standard technique in a blinded, placebo-controlled, randomized fashion. Our study results are consistent with previous studies showing the relative equivalence of desflurane and propofol. ${ }^{9,19}$ Sloan et a ${ }^{9}$ conducted a retrospective review of 156 spine cases and found no statistically significant differences in cortical SSEP or tcMEP amplitudes, tcMEP stimulation voltages or in the average trial to trial amplitude variability. Similarly, Holdefer et $\mathrm{al}^{19}$ conducted a retrospective study to compare the effects of propofol and desflurane anesthesia on tcMEPs from pediatric patients undergoing spine surgery and observed that MEPs from the thenar eminence and abductor hallucis were comparable during maintenance anesthesia on desflurane (0.6-0.8 MAC) or propofol infusion $(150-300 \mu \mathrm{g} / \mathrm{kg} / \mathrm{min})$. Mean peak-to-peak amplitudes of MEPs under desflurane anesthesia from the thenar eminence and abductor hallucis, respectively, were not significantly different from those under propofol. Stimulation was greater and trains were slightly longer in the desflurane compared with the propofol group. In our study, we did not find any statistically significant difference in stimulation voltage between the groups. However, the voltages required in group $S$ were considerably lower than those in other groups. Interestingly, it is noteworthy to mention that there was higher voltage requirement for group $P$ as compared with group $\mathrm{D}$, which probably relates to the synergistic effect of propofol and dexmedtomidine. Lower BIS scores were also noted in this group, which substantiate the synergistic action. Both these studies used a retrospective, study design, which is a major difference from our study design. In contrast to our findings Malcharek et al observed decreased amplitudes in the desflurane group (MAC 0.5-0.6) compared with the propofol group. ${ }^{12}$ In this prospective study, they evaluated the differences in tcMEP amplitudes between desflurane/remifentanil and propofol/remifentanil in patients without pre-existing motor deficits (PMDs) undergoing carotid endarterectomy (CEA) and concluded that desflurane reduced the tcMEP amplitude more significantly than propofol in patients without PMDs. They also concluded that in patients with initially small amplitudes, desflurane could limit tcMEP recording because it produced a remarkable amplitude reduction, even in patients without PMDs. Variability of amplitudes from baseline was more noticed in the propofol-dexmedetomidine group, but a more stable set of recordings was possible with desflurane and standard groups, which differ from the belief that reduction in amplitude was more noticed in volatile agents and hence to offset this more stimulation intensity was needed. ${ }^{20}$ We did not observe any change in stimulation voltage between the groups to obtain a baseline response. Once baseline response was obtained, we did not alter the stimulation characteristics in any patients unless drop in amplitudes were more than $80 \%$ from the baseline values.

We also observed a decrease in the tcMEP amplitude of brachioradialis in group P at 60 and 90 minutes from the baseline, which was statistically significant. The possible explanation for this might be the theory of "anesthetic fade," which was proposed to justify the suppression in the amplitude after prolonged exposure to anesthesia. ${ }^{21}$ In the study done by Lyon et al, the authors suggested that prolonged exposure to anesthetic agents necessitated higher stimulating thresholds to elicit MEP responses, separate from the dose-dependent depressant effect. Myelopathic patients demonstrated a higher rate of increase in the voltage threshold versus normal subjects..$^{21}$ In our study, such a decremental trend was observed in group P in early 
periods, which might be attributable to the synergistic effect of propofol and dexmedetomidine on muscle evoked potential amplitudes. This finding is further strengthened by observing no such pattern in amplitudes in group $S$ in which dexmedetomidine was not used. However, we did not observe any change in amplitude in the lower limbs. We noticed a decrease in MAP values in group D, which was probably due to the vasodilator effects of volatile agents. A lower value of BIS values in group P compared with other two groups was noticed, which could be explained by the synergistic effect of propofol and dexmedetomidine on BIS values. However, these changes in MAP and BIS did not hamper our recordings in TcMEP.

The major limitation to our study was the small sample size, which could have resulted in subtle differences that probably were not of clinical significance. Second, we did not use train of four (TOF) monitoring to objectify the adequacy of recovery from the intubating dose of rocuronium before TcMEP. Third, we had selected $80 \%$ decrease in the amplitude in motor response as a warning signal for surgeons, which could have led to a variation noted in group P. However, we still obtained stable and reliable amplitudes in group D.

\section{Conclusion}

From our study, we observed that both regimens were found to affect the MEP similarly compared with control groups although statistical significance could not be found. Hence desflurane-deemed combination could be considered an alternative for propofol-dexmedetomidine combination for MEP monitoring.

\section{Conflict of Interest}

None declared.

\section{References}

1 Hadley MN, Shank CD, Rozzelle CJ, Walters BC. Guidelines for the use of electrophysiological monitoring for surgery of the human spinal column and spinal cord. Neurosurgery 2017;81(5):713-732

2 Sloan TB. Evoked potential monitoring. Int Anesthesiol Clin 1996;34(3):109-136

3 Sloan TB, Janik D, Jameson L. Multimodality monitoring of the central nervous system using motor-evoked potentials. Curr Opin Anaesthesiol 2008;21(5):560-564

4 Chen X, Sterio D, Ming X, et al. Success rate of motor evoked potentials for intraoperative neurophysiologic monitoring: effects of age, lesion location, and preoperative neurologic deficits. J Clin Neurophysiol 2007;24(3):281-285

5 Deletis V, Kiprovski K, Morota N. The influence of halothane, enflurane, and isoflurane on motor evoked potentials. Neurosurgery 1993;33(1):173-174

6 Pelosi L, Stevenson M, Hobbs GJ, Jardine A, Webb JK. Intraoperative motor evoked potentials to transcranial electrical stimulation during two anaesthetic regimens. Clin Neurophysiol 2001;112(6):1076-1087

7 Lotto ML, Banoub M, Schubert A. Effects of anesthetic agents and physiologic changes on intraoperative motor evoked potentials. J Neurosurg Anesthesiol 2004;16(1):32-42

8 Clapcich AJ, Emerson RG, Roye DP Jr. et al. The effects of propofol, small-dose isoflurane, and nitrous oxide on cortical somatosensory evoked potential and bispectral index monitoring in adolescents undergoing spinal fusion. Anesth Analg 2004;99(5):1334-1340

9 Sloan TB, Toleikis JR, Toleikis SC, Koht A. Intraoperative neurophysiological monitoring during spine surgery with total intravenous anesthesia or balanced anesthesia with 3\% desflurane. J Clin Monit Comput 2015;29(1):77-85

10 Hernández-Palazón J, Izura V, Fuentes-García D, Piqueras-Pérez C, Doménech-Asensi P, Falcón-Araña L. Comparison of the effects of propofol and sevoflurane combined with remifentanil on transcranial electric motor-evoked and somatosensory-evoked potential monitoring during brainstem surgery. J Neurosurg Anesthesiol 2015;27(4):282-288

11 Chong CT, Manninen P, Sivanaser V, Subramanyam R, Lu N, Venkatraghavan L. Direct comparison of the effect of desflurane and sevoflurane on intraoperative motor-evoked potentials monitoring. J Neurosurg Anesthesiol 2014;26(4):306-312

12 Malcharek MJ, Loeffler S, Schiefer D, et al. Transcranial motor evoked potentials during anesthesia with desflurane versus propofol-a prospective randomized trial. Clin Neurophysiol 2015;126(9):1825-1832

13 Sloan TB, Mongan P, Lyda C, Koht A. Lidocaine infusion adjunct to total intravenous anesthesia reduces the total dose of propofol during intraoperative neurophysiological monitoring. J Clin Monit Comput 2014;28(2):139-147

14 Bala E, Sessler DI, Nair DR, McLain R, Dalton JE, Farag E. Motor and somatosensory evoked potentials are well maintained in patients given dexmedetomidine during spine surgery. Anesthesiology 2008;109(3):417-425

15 Rozet I, Metzner J, Brown M, et al. Dexmedetomidine does not affect evoked potentials during spine surgery. Anesth Analg 2015;121(2):492-501

16 Sloan T. Anesthesia and intraoperative neurophysiological monitoring in children. Childs Nerv Syst 2010;26(2):227-235

17 Mahmoud M, Sadhasivam S, Salisbury S, et al. Susceptibility of transcranial electric motor-evoked potentials to varying targeted blood levels of dexmedetomidine during spine surgery. Anesthesiology 2010;112(6):1364-1373

18 Liu Q, Wang Q Liu H, Wu WKK, Chan MTV. Warning criteria for intraoperative neurophysiologic monitoring. Curr Opin Anaesthesiol 2017;30(5):557-562

19 Holdefer RN, Anderson C, Furman M, Sangare Y, Slimp JC. A comparison of the effects of desflurane versus propofol on transcranial motor-evoked potentials in pediatric patients. Childs Nerv Syst 2014;30(12):2103-2108

20 Shida Y, Shida C, Hiratsuka N, Kaji K, Ogata J. High-frequency stimulation restored motor-evoked potentials to the baseline level in the upper extremities but not in the lower extremities under sevoflurane anesthesia in spine surgery. J Neurosurg Anesthesiol 2012;24(2):113-120

21 Lyon R, Feiner J, Lieberman JA. Progressive suppression of motor evoked potentials during general anesthesia: the phenomenon of "anesthetic fade." J Neurosurg Anesthesiol 2005;17(1):13-19 